\title{
Influence of superlattice produced by coherent light beams on the optical properties of ferromagnetic semiconductors
}

\author{
O. Yu. Semchuk, V. V. Gozhenko \\ Institute of Surface Chemistry, National Academy of Sciences of Ukraine \\ 31, prospect Nauki, 252022 Kyiv, Ukraine
}

\begin{abstract}
In this work we study influence of the periodical nanostructures - superlattices, produced by coherent light beams on the optical phenomena in ferromagnetic semiconductors (FMSC). It is shown that as a result of the incident electromagnetic wave diffraction on the periodical nanostructure-superlattice of electron density two waves appear in which periodic energy transfer from one wave to another takes place. We have determined the light reflection coefficient for the surface of FMSC with periodical nanostructures-superlattices. It is also shown that varying a magnitude of the external electric field one can decrease this coefficient, i.e. make FMSC «antirefractive», as well as increase it up to the case of the total reflection.
\end{abstract}

Keywords: superlattice, nanostructure, coherent light beams, electromagnetic wave, ferromagnetic semiconductor, reflection, surface.

Paper received 16.09.99; revised manuscript received 28.09.99; accepted for publication 04.10.99.

\section{Introduction}

Recently, semiconductors with the spatial periodic nanostructures - superlattices have been a subject of intensive studies. Firstly, in connection with appearance of high-power nano- and picosecond lasers as well as corresponding recording facilities the experimental studies of such structures have considerably grown. Secondly, there has been a significant increase of interest in different spatial periodcal nanostructures in view of intensive development of electronics, holography, and semiconductor technology. The FMSC with periodic spatial nanosructures - superlattices on the nonequilibrium electrons and magnons may become the objects, which completely meet the case. Superlattices in FMSC can be formed using coherent light beams (CLBs). For the first time such FMSC with the superlattice of nonequilibrium electrons and magnons were described in $[1,2]$.

In this work we considere influence of CLB's on the optical properties of FMSC. It is shown that two waves appear as a result of the incident electromagnetic wave diffraction on the superlattice on electron density and the periodic energy transfer from one wave to another takes place. We have determined the light reflection coefficient for the outer surface of FMSC with the periodic nanostruc-ture superlattice of electron density.

\section{FMSC with superlattices}

Let us consider a wide-gap FMSC of the EuO-type with an average carrier density $n_{0}$ in the external constant electric field $\vec{F}_{0} \| O Z$ at the spin-wave temperature. Let several CLB be incident on the outer surface $x=0$ of the FMSC. The vector-potential of the CLB in the material is given by:

$\vec{A}(\vec{r}, t)=\sum_{j} \vec{A}_{j} \cos \left(\omega t-\vec{k}_{j} \vec{r}-\varphi_{j}\right)$,

and their frequency $\omega$ satisfies the inequality $\bar{\varepsilon}<<\omega<<\varepsilon_{g}$ ( $\bar{\varepsilon}$ is the average carrier energy, $\varepsilon_{g}$ is the energy-gap width). Therefore, the CLB does not change a total number of carriers and only causes their spatial redistribution. A nonequilibrium state in the FMSC is produced by heatingup electrons in the field $\vec{F}_{0} \| O Z$ and as a result of light absorption by free carriers, the consequent energy transfer from electrons to magnons takes peace. The phonons play a role of thermostat. Spatial modulation of the collision integrals and high-frequency CLB wave pressure on electrons leads to the formation of the periodical nanostructures - superlattices of carrier density $n$, electron $\Theta_{e}$ and magnon $\Theta_{m}$ temperatures, electric field strength $\vec{F}$, and nonequilibrium magnetization $M$ of FMSC [1,2]. In the case of incidentce on the FMSC surface of only two symmetrically oriented 


\section{O. Yu. Semchuk, V. V. Gozhenko: Influence of superlattice produced by...}

beams with $\vec{A}_{1} \| \vec{A}_{2}, \quad k_{1 x}=k_{2 x}, k_{1 z}=-k_{2 z}$, these periodic structures can be described by the following expression:

$$
\begin{aligned}
& n(z)=n_{0}\left(1+\xi_{1} \cos 2 k_{1 z} z+\xi_{2} \sin 2 k_{1 z} z\right), \\
& F(z)=F_{0}\left(1+\varsigma_{1} \cos 2 k_{1 z} z+\varsigma_{2} \sin 2 k_{1 z} z\right), \\
& \Theta_{e}=T_{e}\left(1+\eta_{0}+\eta_{1} \cos 2 k_{1 z} z+\eta_{2} \sin 2 k_{1 z} z\right), \\
& \Theta_{m}=T_{m}\left(1+\mu_{0}+\mu_{1} \cos 2 k_{1 z} z+\mu_{2} \sin 2 k_{1 z} z\right) .
\end{aligned}
$$

The temperatures $T_{e}, T_{m}$ in the field $F_{0}$ are determined by the familiar technique and will be further regarded as known quantities. The amplitudes $\xi_{i}, \varsigma_{i}, \eta_{i}, \mu_{i}$ depend on $A_{1}, A_{2}$, $F_{0}$ and FMSC parameters; the method of their calculation is given in general form in our previous papers $[1,2]$. We present here only the expression for amplitudes $\xi_{1}$ and $\xi_{2}$ since, as it will be seen from the subsequent calculations, these define the processes of wave diffraction and scattering in FMSC with the periodical nanostructure - superlattice:

$$
\xi_{1}=\frac{1}{(\alpha+1)^{2}+\beta^{2}}\left\{\begin{array}{l}
\frac{\beta^{2}-\alpha-1}{2} \eta_{1}+\beta(\alpha+1) \eta_{2}+2 \beta^{2} \mu_{1}+ \\
2 \beta(\alpha+1) \mu_{2}+\frac{\beta^{2} B}{2}\left[\frac{1}{3}\left(\frac{\pi T_{e}}{\hbar \omega}\right)^{\frac{1}{2}}-\frac{\alpha+1}{\beta}\right]
\end{array}\right],
$$

$\xi_{2}=\frac{1}{(\alpha+1)^{2}+\beta^{2}}\left\{\begin{array}{l}-\beta \frac{2 \alpha+1}{2} \eta_{1}-\frac{\alpha+1-\beta^{2}}{2} \eta_{2}-2 \beta(\alpha+1) \mu_{1} \\ -2 \beta^{2} \mu_{2}-\frac{\beta^{2} B}{2}\left[\frac{\alpha+1}{3}\left(\frac{\pi N_{e}}{\hbar \omega}\right)^{\frac{1}{2}}-1\right]\end{array}\right\}$

where $\alpha=\frac{\pi e^{2} n_{0}}{\varepsilon_{0} k_{1 z}^{2} T_{e}}, \beta=\frac{e F_{0}}{2 k_{1 z} T_{e}}, B=\frac{4 e A_{1} A_{2}}{3 \beta m c^{2} T_{e}}, \varepsilon_{0}$ - is the relative permitivity of FMSC, $m$ is the electron effective mass.

\section{Light diffraction in FMSC by periodical nanostructures}

Let a weak electromagnetic wave with polarization, which differs from that of CLB, propagates in FMSC along $\mathrm{O} x$ axis. We suppose for simplicity that the wave amplitude depends on the coordinate $z$, and given by usual formula

$$
E=E(x) \exp \left[i\left(k_{x} x+k_{1 z} z-\Omega t\right)\right],
$$

and frequency $\Omega$ is considerably higher than that of electron collisions.

The current density $j$ induced by the wave (5) in linear approximation is given by expression $j=\frac{i e^{2} n_{0}}{m \Omega}\left[1+\frac{n_{1}(z)}{n_{0}}\right] E$.

Substituting (6) into the Maxwell equations for the wave (5), we obtain

$$
\begin{aligned}
\nabla^{2} E & +\frac{\varepsilon_{0}}{c^{2}}-\left(\Omega^{2}-\omega_{p}^{2}\right) E= \\
= & \alpha_{1}\left(\xi^{*} \exp \left(2 i k_{1 z} z\right)+\xi \exp \left(-2 i k_{1 z} z\right)\right) E
\end{aligned}
$$

where $\omega_{p}^{2}=4 \pi n_{0} e^{2} / \varepsilon_{0} m, \alpha_{1}=\varepsilon_{0} \omega_{p}^{2} / 2 c^{2}, \xi^{*}=\xi_{1}+i \xi_{2}$. We consider a solution of (7) in the following form $E=\sum_{l} E_{l}(x) \exp \left\{i\left(k_{x} x+k_{1 z} z\right)\right\}$ with the boundary conditions $E(0)=E^{(0)}, E_{l=0}^{(0)}=0$. Since in our case $|\xi|<<1$, the most important terms in the general expression for $E$ corresponding $l=0$ and $l=-1$. Moreover, smallness of the parameter $|\xi|$ value enables us to obtain the following set of equations

$2 i k_{x} \frac{d E_{0}}{d x}=\alpha_{1} \xi^{*} E_{-1}, 2 i k_{x} \frac{d E_{-1}}{d x}=\alpha_{1} \xi E_{0}$,

They have a simple solution satisfying the boundary conditions:

$$
\begin{aligned}
& E_{0}=E^{(0)} \cos \left(\alpha_{1}^{2}|\xi| / 2 k_{x}\right) x, \\
& E_{-1}=-i \frac{|\xi|}{\xi^{*}} E^{(0)} \sin \left(\alpha_{1}^{2}|\xi| / 2 k_{x}\right) x .
\end{aligned}
$$

The solution (9) defines two waves, which arise as a result of diffraction of incident wave on the periodical nanostructure - superlattice of electron density, which turns out to be a periodic function of $x$-coordinate .

Thus, two waves arise and the periodic energy transfer from one wave to another takes place as a result of diffracting incident electromagnetic waves on a periodical nanostructure - superlattice of electron density.

\section{Electromagnetic wave scattering by periodical nanostructures}

Now, let us consider the electromagnetic wave reflection from the surface $z=0$ FMSC by periodical nanostructuressuperlattices. We determine the electric field of the incident wave in vacuum as:

$$
E=E^{(0)}\left[\exp \left\{i\left(k_{x} x+u_{z} z\right)\right\}+R_{0} \exp \left\{i\left(k_{x} x-u_{z} z\right)\right\}\right] \exp (-i \Omega t)
$$

where, $\Omega^{2}=c^{2}\left(k_{x}^{2}+u_{z}^{2}\right), u_{z}^{2}=\left(\omega_{p}^{2} / c^{2}\right)+\varepsilon_{0}^{-1} \mid k_{x}^{2}-$ $\left.\left(\varepsilon_{0}-1\right) S_{z}^{2}\right], S_{z}=k_{1 z}+\Delta_{k}$

The calculation gives the following expression for the reflection coefficient in the Bragg resonance region: 
$\left|R_{0}\right|^{2}=\frac{2 k_{1 z}\left(u_{z}^{2}+k_{1 z}^{2}\right) \Delta_{k}-4 u_{z} k_{1 z}^{2} \delta-\alpha_{1}\left(k_{1 z}^{2}-u_{z}^{2}\right) \xi_{1}}{2 k_{1 z}\left(u_{z}^{2}+k_{1 z}^{2}\right) \Delta_{k}+4 u_{z} k_{1 z}^{2} \delta-\alpha_{1}\left(k_{1 z}^{2}-u_{z}^{2}\right) \xi_{1}}$,

where $\delta= \pm \sqrt{\Delta_{k}^{2}-\alpha_{1}^{2}\left(\xi_{1}^{2}-\xi_{2}^{2}\right) / 4 k_{1 z}^{2}}$.

Figure 1 shows the dependence of the reflection coefficient on the parameter $\Delta_{k}$ for the outer surface of EuO. When calculating the curve we used the following parameters $n=10^{19} \mathrm{sm}^{-1}, \omega=5 \cdot 10^{15} \mathrm{~s}^{-1}, T=0.5 \cdot 10^{-21} \mathrm{~J}, F_{0}=2 \cdot 10^{3} \mathrm{~V} / \mathrm{sm}$. The reflection coefficient decreases and reaches a minimum which is equal to $2.547 \cdot 10^{-4}$ at $\Delta_{k}=-0.21$.

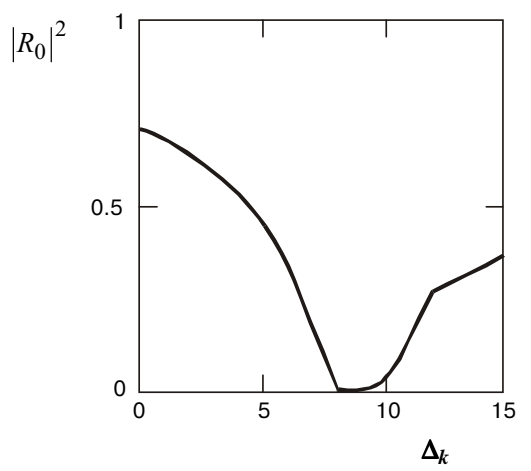

Fig.1. The dependence $\left|R_{0}\right|^{2}$ on $\Delta_{k}$.

\section{Conclusions}

We have carried out a study of the optical phenomena in FMSC with a periodical nanostructures - superlattices of nonequilibrium electrons and magnons. The results of investigations enable us to conclude the following:

1. As a result of diffraction of electromagnetic waves on the periodical nanostructure of electron density in FMSC, we obtain two waves propagate along $\mathrm{O} x$-axis appear. The periodic energy transfer from one wave to another as for they penetrate from a surface into the bulk of FMSC takes place.

2. The reflection coefficient of electromagnetic wave for the outer surface $z=0$ of FMSC under conditions of the Bragg resonance depends indirectly on the values of the heating field, angle of incidence and the parameters of FMSC. Varying those and the value of $F_{0}$, one can decrease the reflection coefficient, i.e. make FMSC the «antirefractive», as well as increase it, reaching the case of almost total reflection of the electromagnetic wave from the surface of FMSC.

\section{References}

1. Semchuk A.Yu., Levshin A.E. and Tomchuk P.M., Sov.Phys.Solid State, 1986, 28, 229.

2. Semchuk A.Yu., Grechko L.G., and Ogenko V.M., Phys.stat.sol(b), 1990, 157, 451. 